\title{
Disappointing outcomes for anti-VEGF therapy
}

Two new studies have reported negative outcomes in patients with cancer treated with anti-VEGF agents. The results suggest that, in pancreatic cancer, targeting of VEGF signaling is an ineffective strategy, and more worryingly, the drug bevacizumab might be associated with an increased risk for treatment-related death.

\section{4 ...bevacizumab significantly increased the risk of fatal toxicities in cancer patients... 77}

In a randomized, double-blind, phase III study, Hedy Kindler and co-workers investigated the effect of adding axitinib to gemcitabine for the treatment of advanced pancreatic cancer. "Axitinib is a potent and selective inhibitor of VEGF receptors 1,2, and 3 that has demonstrated clinical efficacy in multiple solid tumors," Kindler describes. No significant difference in overall survival was observed between patients receiving gemcitabine alone or in combination with axitinib (8.3 months versus 8.5 months).

"These data do not support results from the earlier phase II study suggesting that axitinib may improve survival when combined with gemcitabine in this setting," comments Kindler. The data do, however, support findings from other studies of bevacizumab and aflibercept suggesting that inhibition of the VEGF pathway is ineffective in patients with pancreatic cancer. "The results highlight the importance of conducting phase III evaluation only after detection of a robust signal from appropriately designed phase II studies," Kindler adds.

Ranpura and colleagues performed a meta-analysis of 16 randomized controlled trials that investigated bevacizumab therapy in a range of cancers. Their aim was to determine the risk of fatal adverse events associated with bevacizumab treatment. When added to chemotherapy or biological therapy, "bevacizumab significantly increased the risk of fatal toxicities in cancer patients," explains senior researcher Shenhong Wu. The overall incidence of fatal adverse events with bevacizumab in combination with chemotherapy was $2.5 \%$ compared with $1.7 \%$ in patients who received chemotherapy alone.

These findings add to a growing body of evidence that sugges the potential benefits of treatment with bevacizumab should be weighed against the risk of fatal adverse effects. Wu's team plan further investigations to identify risk factors for fatal toxic effects that could help select suitable patients for bevacizumab treatment. "Overall survival instead of progression-free survival should be the primary end point for future bevacizumab-based studies," concludes Wu.

Lisa Richards

Original articles Kindler, H. L. et al. Axitinib plus gemcitabine versus placebo plus gemcitabine in patients with advanced pancreatic adenocarcinoma: a double-blind randomised phase 3 study. Lancet Oncol. doi:10.1016/ S1470-2045(11)70004-3 | Ranpura, V et al. Treatmentrelated mortality with bevacizumab in cancer patients: a meta-analysis. JAMA 305, 487-494 (2011) 